\title{
UAB
}

Universitat Autònoma

de Barcelona

This is the accepted version of the article:

Serna, Naroa; Cano Garrido, Olivia; Sanchez, Julieta M.; [et al.]. «Release of functional fibroblast growth factor-2 from artificial inclusion bodies». Journal of Controlled Release, Vol. 327 (Nov. 2020), p. 61-69. DOI 10.1016/j.jconrel.2020.08.007

This version is available at https://ddd.uab.cat/record/233721

under the terms of the (c) BY-NC-ND license 


\section{Release of functional fibroblast growth factor-2 from artificial inclusion bodies}

Naroa Serna ${ }^{1,2,3}$, Olivia Cano-Garrido ${ }^{1,2,3}$, Julieta M. Sánchez ${ }^{1,2,3,4}$, Alejandro SánchezChardi ${ }^{5,6}$, Laura Sánchez-García ${ }^{1,2,3}$, Hèctor López-Laguna 1,2,3 , Ester Fernández ${ }^{7}$, Esther Vázquez ${ }^{1,2,3}{ }^{*}$, and Antonio Villaverde ${ }^{1,2,3 *}$

1 Institut de Biotecnologia i de Biomedicina, Universitat Autònoma de Barcelona, Bellaterra, 08193 Barcelona, Spain

${ }^{2}$ Departament de Genètica i de Microbiologia, Universitat Autònoma de Barcelona, Bellaterra, 08193 Barcelona, Spain

${ }^{3}$ CIBER de Bioingeniería, Biomateriales y Nanomedicina (CIBER-BBN), Spain

${ }^{4}$ Instituto de Investigaciones Biológicas y Tecnológicas (IIBYT) (CONICET-Universidad Nacional de Córdoba) ICTA \& Cátedra de Química Biológica Departamento de Química FCEFyN, UNC. Av. Velez Sarsfield 1611, X 5016GCA, Córdoba, Argentina

5 Servei de Microscòpia, Universitat Autònoma de Barcelona, Bellaterra, 08193 Barcelona, Spain

${ }^{6}$ Departament de Biologia Evolutiva, Ecologia i Ciències Ambientals, Facultat de Biologia, Universitat de Barcelona, Av. Diagonal 643, 08028 Barcelona, Spain

${ }^{7}$ Departament de Biologia Cel·lular, Fisiologia i Immunologia . Universitat Autònoma de Barcelona, Bellaterra, 08193 Barcelona, Spain

¥ Present address; Nanoligent SL. Edifici Eureka, Universitat Autònoma de Barcelona, Bellaterra, 08193 Barcelona, Spain

*Corresponding authors. EV esther.vazquez@uab.cat; AV Antoni.Villaverde@uab.cat Keywords: secretory granules; protein materials; extended drug release; wound healing; hFGF-2 


\begin{abstract}
Growth factors are required for cell proliferation and differentiation under physiological conditions but especially in the context of regenerative medicine. The time-prolonged administration of those factors has been explored using different sustained drug delivery systems. These platforms include natural materials such as bacterial inclusion bodies (IBs) that contain chaperones and other bacterial components that might favour protein release. Being successful from a functional point of view, IBs pose regulatory concerns to clinical applications because of the mentioned presence of bacterial cell components, including endotoxins. We have here explored the release and activity of the human fibroblast growth factor-2 (hFGF-2) from a novel synthetic material, namely artificial IBs. Being chemically homogenous and compliant with regulatory restrictions, we wondered if these materials would effectively release functional proteins in absence of accompanying bacterial agents. The data provided here fully supports that artificial hFGF-2 IBs act as true and efficient secretory granules and they slowly disintegrate in cell culture to promote wound healing in an in vitro wound healing model. Free from undesired bacterial components, artificial inclusion bodies show promises as delivery agents in regenerative medicine.
\end{abstract}




\section{Introduction}

Delivery systems for therapeutic proteins are required in different clinical fields, to avoid or delay repeated protein dosing that might become inconvenient or unreachable. Protein replacement therapies and especially regenerative medicine are among the main clinical targets for protein delivery systems. Many strategies are based on the design of biocompatible matrices or networks to be used as protein holders. Built with organic or inorganic composites, these structures pursue a regulatable and timesustained release of the embedded protein in absence of toxicities linked to the material forming the matrix [1-6].

In this context, the secretory granules of the human endocrine system, that release peptidic hormones, are of particular interest. These structures are proteinaceous materials in which the protein components themselves are physiologically released by a slow disintegration process [7]. This is based on a particular architecture of such secretory agents, which are in fact functional amyloids $[8,9]$. From them, the building block itself, that is, the protein hormone, is slowly released by a process that involves molecular chaperones [7]. Mimetics of those secretory materials would be then very useful in biomedicine, as their use would not require any heterologous matrix or supporting scaffold to release the functional protein. In this regard, bacterial inclusion bodies (IBs), that are micro-scale protein aggregates formed in recombinant bacteria by the product of the cloned gene, behave, in many aspects, like those secretory granules [10]. Being intriguing protein depots [11], IBs have been tailored as a source of functional proteins in cell culture, to release growth factors [12, 13], chaperones [13], cytoskeleton components [14] and anti-oxidant proteins [13]. In two different mouse models of human cancers, the local or remote administration of IBs formed by antitumoral proteins show potent antitumoral [15] and antimetastatic effects [16] respectively. Being structural and functional mimetics of secretory granules, IBs show promise as protein delivery systems [17], but their bacterial origin imposes the presence of molecular contaminants and endotoxins whose full elimination is unapproachable by industrially compliant methods. Therefore, the use of IBs results non-appropriate for most of clinical applications. Very recently [18], micron sized artificial IBs (ArtIBs) have been generated by very simple in vitro fabrication approaches, using divalent ions as 
molecular cross-linkers and a functional protein as a unique building block. If efficiently releasing the forming protein, such ArtIBs might represent better mimetics of secretory granules than IBs. Being fabricated out of pure protein, they are chemically homogeneous and with a defined composition, what is highly appealing in clinics. However, since they are generated in vitro by the controlled assembly of one single protein species and free from any bacterial component, they are also devoid of chaperones from the bacterial quality system that are associated to natural IBs [19]. Whether IB-associated bacterial chaperones are necessary for the release of functional protein from these functional amyloids has been not yet determined. Therefore, the ability of ArtIBs to deliver functional growth factors (and other clinically relevant proteins) should be assessed before further development and even before any detailed in vivo analyses of functionality. In this context, we have designed, fabricated and fully characterized ArtlBs formed by the hFGF-2 in an in vitro wound healing model, and evaluated the potential of these materials as functional protein depots for a timeprolonged healing effect.

\section{Materials and methods}

Plasmid, culture \& protein production

The synthetic hFGF-2-H6 gene (Low Molecular Weight isoform; $18 \mathrm{KDa}$ ) and the control GFP-H6 gene were designed in house to be inserted into the prokaryotic expression vector $\mathrm{pET} 22 \mathrm{~b}$ and obtained from GeneArt (Thermo Fisher Scientific). The amino acid sequences of the encoded proteins hFGF-2-H6 and GFP-H6 are MAAGSITTLPALPEDGGSGAFPPGHFKDPKRLYCKNGGFFLRIHPDGRVDGVREKSDPHIKLQLQA EERGVVSIKGVCANRYLAMKEDGRLLASKCVTDECFFFERLESNNYNTYRSRKYTSWYVALKRTGQ YKLGSKTGPGQKAILFLPMSAKSHHHHHH and MSKGEELFTGVVPILVELDGDVNGHKFSVSGEGEGDATYGKLTLKFICTTGKLPVPWPTLVTTLTYG VQCFSRYPDHMKRHDFFKSAMPEGYVQERTISFKDDGNYKTRAEVKFGDTLVNRIELKGIDFKED GNILGHKLEYNYNSHNVYITADKQKNGIKANFKIRHNIEDGSVQLADHYQQNTPIGDGPVLLPDN HYLSTQSALSKDPNEKRDHMVLLEFVTAAGITHGMDELYKHHHHHH), respectively. H6 stands 
for the carboxy-hexahistidine tail, which simultaneously serves for protein purification, for divalent cation coordination and for ArtIB fabrication.

The Escherichia coli (E. coli) strain BL21 (DE3) was used for the production of bacterial IBs and soluble protein, once transformed by heat shock with the recombinant $\mathrm{pET} 22 \mathrm{~b}$ vectors. The $E$. coli cultures were kept at $37{ }^{\circ} \mathrm{C}$ and $250 \mathrm{rpm}$ up to $0.5 \mathrm{OD}$ units. In order to promote the protein deposition as bacterial IBs, gene expression was induced for $3 \mathrm{~h}$ at $37{ }^{\circ} \mathrm{C}$ upon isopropyl-D-thiogalactoside (IPTG) at $1 \mathrm{mM}$. For soluble protein production, cells were cultured overnight at $20^{\circ} \mathrm{C}$ and the gene expression was induced with IPTG at $0.1 \mathrm{mM}$. Then, bacterial cells were harvested by centrifugation $(5,000 \mathrm{~g}$ at $4{ }^{\circ} \mathrm{C}, 15 \mathrm{~min}$ ) and freezed at $-80^{\circ} \mathrm{C}$.

\section{Bacterial IBs and ArtIBs fabrication}

Bacterial hFGF-2-H6 IBs were isolated by a combination of enzymatic and soft mechanical cell lysis by a protocol described elsewhere [20]. IB proteins were quantified by Western Blot using an anti-His antibody (1:5000 Anti-His Santa Cruz Biotechnology, ref: sc-57598). Sample protein amounts were inferred from standard curves with known amounts of hFGF-2-H6 protein using the Quantity One software.

hFGF-2-H6 and non-functional GFP-H6 ArtIBs were fabricated from pure soluble proteins. For purification of these soluble recombinant proteins, bacterial cells were resuspended in wash buffer ( $20 \mathrm{mM}$ Tris- $\mathrm{HCl}, 500 \mathrm{mM} \mathrm{NaCl}$ and $10 \mathrm{mM}$ imidazole $\mathrm{pH}$ 8), containing an EDTA-free protease inhibitor cocktail (Roche). Afterwards, E. coli cells were disrupted by three rounds at 1200 psi in a French press (Thermo FA-078A). Both proteins hFGF-2-H6 and GFP-H6 were purified by His-tag affinity chromatography using $1 \mathrm{ml}$ HiTrap Chelating HP columns (GE Healthcare) through an ÄKTA pure FPLC (GE Healthcare). Fractionation was made by a linear gradient of elution buffer ( 20 mM Tris$\mathrm{HCl}, 500 \mathrm{mM} \mathrm{NaCl}$ and $500 \mathrm{mM}$ imidazole $\mathrm{pH}$ 8). Purified protein fractions were dialyzed against carbonate buffer $\left(166 \mathrm{mM} \mathrm{NaCO}{ }_{3} \mathrm{H}, \mathrm{pH}\right.$ 8). Soluble protein was then stain-free detected by TGX (TGX FastCast Acrylamide Kit) technology and protein amounts were pure soluble proteins in distilled $\mathrm{H}_{2} \mathrm{O}$ at a final concentration of $2 \mathrm{mg} \mathrm{mL}^{-1}$ and a finalv 
volume of $200 \mu \mathrm{L}$. Afterwards, protein samples were mixed by default with $\mathrm{ZnCl}_{2}$, at a zinc:protein molar ratio of 50:1 for hFGF-2 ArtlBs and at 100:1 for GFP ArtIBs (1:1 ratio stands for $0.196 \mathrm{mM}$ ). Then, samples were incubated $10 \mathrm{~min}$ at room temperature and centrifuged at $15.000 \mathrm{~g}$ vfor $15 \mathrm{~min}$. Finally, the soluble fraction was discarded to obtain the final product. The discarded soluble protein was quantified by Bradford assay to determine the exact concentration of artificial inclusion bodies that correspond to the remaining protein. Alternatively, other divalent cations namely $\mathrm{CaCl}_{2}$ and $\mathrm{MnCl}_{2}$ were used at cation:protein molar ratio of 200:1 and 400:1 for hFGF-2 ArtIB, and at 300:1 and 500:1for the control GFP ArtIBs formation.

\section{Ultrastructural characterization}

Volume size distribution of bacterial IBs and ArtIBs was determined by dynamic light scattering (DLS) at $633 \mathrm{~nm}$ in a Zetasizer Nano ZS (Malvern Instruments Limited) using ZEN2112 $3 \mathrm{~mm}$ quartz batch cuvettes. Protein samples dissolved in distilled $\mathrm{H}_{2} \mathrm{O}$ at $1 \mathrm{mg}$ $\mathrm{mL}^{-1}$ were measured in triplicate and mode size peak and polydispersion index (PDI; expressed as mean \pm standard error of the mean) were obtained. $v$

Ultrastructural morphometry (size and shape) of bacterial IBs and ArtIBs was visualized at nearly native state with electron microscopy. Drops of $20 \mu \mathrm{L}$ of IBs resuspended in deionized water were directly deposited on silicon wafers (Ted Pella Inc.) for 2 min, excess of liquid blotted, air dried, and immediately observed without coating in a field emission scanning electron microscope (FESEM) Merlin (Zeiss) operating at $1 \mathrm{kV}$ and equipped with a standard secondary electron detector. Representative images of general fields and nanostructure details were captured at three high magnifications $(10,000 x, 40,000 x$ and $80,000 x)$.

\section{Thioflavin T fluorescence in ArtIBs}

The interaction of Thioflavin T with ArtIBs was explored to evaluate the amyloidal nature of the protein material. Protein aliquots $(30 \mu \mathrm{l})$ were added to $25 \mu \mathrm{M}$ Thioflavin T (Sigma Aldrich) (Thio T) $(30 \mu \mathrm{l})$ in carbonate buffer $\left(166 \mathrm{mM} \mathrm{NaCO}_{3} \mathrm{H}, \mathrm{pH}\right.$ 8). The final protein 
concentration was $0.1 \mathrm{mg} / \mathrm{ml}$. ThioT fluorescence was excited at $450 \mathrm{~nm}$ and the fluorescence emission spectra were recorded with a Varian Cary Eclipse spectrofluorimeter. The cross-beta sheet structure of the hFGF-2 within ArtIBs was monitored by the enhancement of the free dye fluorescence emission caused by the interaction with amyloidogenic proteins.

\section{Soluble protein release}

Bacterial IBs and ArtIBs were resuspended in $1 \mathrm{~mL}$ of PBS reaching a final concentration of $2 \mathrm{mg} \mathrm{mL}^{-1}$ a $\mathrm{nd}$ i $\mathrm{ncu}$ b a $\mathrm{t}$ e d a t $37{ }^{\circ} \mathrm{C}$ wi $\mathrm{th}$ o u each sample at 24, 72, 120 and $168 \mathrm{~h}$ time points and centrifuged for $10 \mathrm{~min}$ at 15,000 $\mathrm{g}$ to separate soluble and insoluble fractions. Then, soluble protein was detected by stain-free TGX (TGX FastCast Acrylamide Kit) and by Western Blot using an anti-His monoclonal antibody (1:5000 Anti-His Santa Cruz Biotechnology, ref: sc-57598). The percentage of released protein was quantified by Image Lab software.

\section{Thermal stability of the released proteins}

Fluorescence spectra were recorded in a Cary Eclipse spectrofluorimeter (Agilent Technologies), using a quartz cell with $2 \mathrm{~mm}$ path length and a thermostated holder. The excitation and emission slits were set at $5 \mathrm{~nm}$. Excitation wavelength ( ex) was set at 295 $\mathrm{nm}$. Emission spectra were acquired between 310 and $450 \mathrm{~nm}$ in hFGF-2 samples at 0.1 $\mathrm{mg} / \mathrm{mL}$, dissolved in carbonate buffer $\left(166 \mathrm{mM} \mathrm{NaCO}_{3} \mathrm{H}, \mathrm{pH}\right.$ 8) upon release from ArtlBs. The temperature-dependent behavior of proteins was determined between 25 and 80 ㅇ․ The Centre of Spectral Mass (CSM), a weighted average of the fluorescence spectrum peaks [21] was calculated for comparison. The CSM is also related with the relative exposure of the tryptophan (Trp) to the protein environment. The maximum red-shift in the CSM of Trp is compatible with large solvent accessibility and unfolding [22]. The protein thermal stability was analysed by the onset temperature ( $\left.T_{\text {onset }}\right)$. This parameter indicate the temperature where the CSM start to increase. 
Biological activity of bacterial IBs, ArtIBs, soluble protein and cations ( $\mathrm{ZnCl}_{2}, \mathrm{CaCl}_{2}$ and $\mathrm{MnCl}_{2}$ ) was analyzed by measuring cell proliferation and survival of the murine cell line NIH3T3. NIH3T3 cells were routinely maintained in D-Minimum Essential Medium Eagle (D-MEM) supplemented with $10 \%$ Fetal Bovine Serum (FBS) and 2 mM L-glutamine at $37^{\circ} \mathrm{C}$ and $10 \% \mathrm{CO}_{2}$ in a humidified incubator.

For proliferation studies, bacterial IBs and ArtIBs were resuspended in cell culture medium with $1 \%$ FBS at different concentrations. ArtIBs formed by the fluorescent protein GFP-H6 were included as negative control and recombinant soluble hFGF-2 was used as a positive control. 96-well plates were decorated with IBs and ArtIBs, adding 50

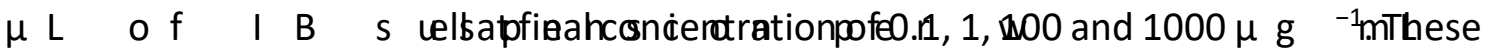
plates were incubated at $4{ }^{\circ} \mathrm{C}$ overnight in order to allow IBs and ArtIBs sedimentation. $4 \times 10^{3}$ NIH3T3 cells were seeded per well and cultured for $48 \mathrm{~h}$ at $37{ }^{\circ} \mathrm{C}$ and $10 \% \mathrm{CO}_{2}$ in a humidified incubator. Cell proliferation was determined by CellTiter-Glo Luminescent Cell Viability Assay (Promega) in a Multilabel Plater Reader Victor3 (Perkin Elmer). All experiments were done with three replicates.

For cell survival assay, 96-well plates were decorated with bacterial IBs and ArtIBs at a final concentrat i o $n \quad \circ \mathrm{f}^{1}$. Th飞en, NuHBT3 cells were seeded at $4 \times 10^{3}$ cells per well a nd cel l survival was measured at different the CellTiter-Glo ${ }^{\circledR}$ Reagent (Promega).

The positive control soluble hFGF-2 was added together with NIH3T3 cells. It was

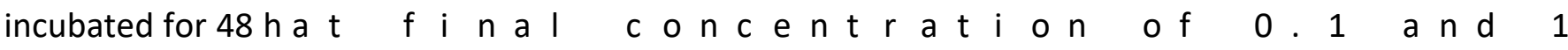
a $\mathrm{n} \mathrm{d} \mathrm{a} \mathrm{t}^{-1} \mathrm{f}$ gr cell sgrvivarl assay. All experimental data were measured in triplicate.

\section{Wound healing assay}

Wound healing assays were performed by decorating the cell culture surface with GFP and hFGF-2 ArtIBs $\mathrm{Zn}^{+2}$ and soluble hFGF-2 protein. Briefly, ArtIBs and soluble protein a I i quots were defrost ed a $\mathrm{nd} r$ es us pended i $n$ added to each of the two compartments of a silicone insert (culture-insert, 2-wells 24- 


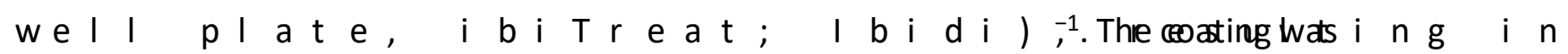
incubated for $4 \mathrm{~h}$ at $4{ }^{\circ} \mathrm{C}$. Then cells were seeded with a cell density of $4 \times 10^{4}$ cells $\mathrm{mL}^{-1}$ and after $24 \mathrm{~h}$ the medium was changed to starvation medium containing only $1 \%$ FBS. Cells were grown further and after $24 \mathrm{~h}$ the culture-inserts were removed using sterile $t$ w e e $z$ e $r$ s $r$ e s u-wide gap. Alore strarvation rheđịm wasmdded to cover the whole well of the 24-well plate. The subsequent healing process was recorded using

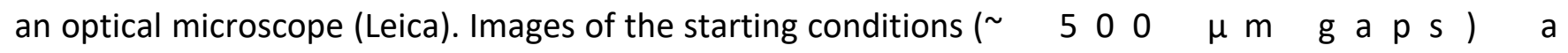
different time points for at least two wells were taken and all wells of the 24-well plate were checked visually for any irregularities. Wound sizes were determined from four independent wells from two plates, using Image J software with the MRI wound healing tool macA hro.

\section{Statistical Analyses}

P a i r wise comparisons of protei $n \quad c$ etestl $v i a b i$ and multiple comparisons to detect differences between control and experimental g r o u ps of $p r \circ$ i f e $r$ a $t$ i o $n$ a $n$ Adl statiscical tests whre a l i $n g$ performed using GraphPad Prism version 8.0. All quantitative values were expressed as mean \pm standard error of the mean $(\bar{x} \pm$ SEM). Differences among groups were considered significant at $p<0.05$ and relevant divergences were marked as * $(p<0.01)$ or \# $(p<0.05)$.

\section{Results and discussion}

A H6-tagged hFGF-2 (Figure 1A, top) was produced in recombinant bacteria under conventional (mild) production conditions or under conditions promoting conformational stress (Figure 1A). Mild conditions favoured the production of soluble protein versions, that upon purification, were further used for ArtlB fabrication (Figure 1A). The purified soluble hFGF-2 was proteolytically stable (Figure $1 B, C$ ), and it showed a tendency to oligomerization (Figure 1C). For protein precipitation as ArtlBs, we used three different divalent cations at the respective molar proportions that lead to full protein precipitation, meaning that remaining soluble protein was undetectable (Figure 
1D). On the other hand, the stress-prone culture conditions (Figure 1A) favoured the formation of bacterial IBs that were isolated and straightforward used in the experimental. Such bacterial IBs exhibited around $600 \mathrm{~nm}$ (Figure 2A), while ArtIBs (those generated with $\mathrm{Zn}^{2+}$ cation and named ArtIBs $\mathrm{Zn}$ ) doubled this size, indicative of a successful His- and Zn-mediated assembly of the His-tagged hFGF-2. GFP-H6 ArtIBs were also fabricated as experimental controls, sizing around $1.8 \mu \mathrm{m}$ (Figure 2A). Morphologically, hFGF-2 IBs and ArtIBs Zn were very similar, showing the typical rough surface of conventional IBs [23]. The purity of ArtIBs, formed by a single immunoreactive protein species, contrasted with the extreme heterogeneity in the protein composition of IBs, as revealed by the broad brand spectrum of these materials (Figure 2C). Such heterogeneity had been previously reported in IBs during early proteomic studies of such materials $[19,24]$, and the presence of different types of bacterial chaperones (holding, folding and disaggregating) also repeatedly confirmed as a part of the IB proteome [19, 24-29]. In addition to the morphological similarity with conventional IBs, the amyloid nature of ArtIBs was evaluated by the interaction of Thioflavin $\mathrm{T}$, showing a significant extent of cross beta secondary structure (Figure 2D) that was in agreement with previous data obtained with different ArtIB species[30].

To start the hFGF-2 ArtIB characterization, we decorated with different types of protein clusters cell culture plates where NIH3T3 were further seeded. Control GFP ArtIBs formed with the assistance of different ions did not show any proliferative effect, and cell growth over them was fully comparable to control cell cultures in absence of additional materials (Figure 3A). However, hFGF-2 ArtIBs clearly stimulated cell proliferation at levels similar to those promoted by the soluble hFGF-2 and slightly higher than those promoted by hFGF-2 bacterial IBs (Figure 3A). Such effect was observed at two different concentrations of the protein material, and the nature of the ion used as cross-linker did not show clear influences on the biological impact of the material (Figure $3 \mathrm{~A}$ ). Then $\mathrm{Zn}$ cation was at this point selected as a default ion for fabrication of the protein granules. The fact that ArtIBs tended to stimulate cell growth with more intensity than IBs, was indicative of a higher bioavailability of hFGF-2 in the artificial materials compared to natural versions, at a similar extent than that offered by 
the plain soluble protein version. At this stage, we wondered how hFGF-2 ArtlBs would behave in a cell survival assay, in which cells were cultured in a starving medium. Again, the biological impact of ArtIBs was fully comparable to that of the soluble protein, since both agents rescued with matching profiles starving cells, which died in a few days in the presence of GFP materials or without any additive (Figure 3B). The rescuing ability of both forms of hFGF-2 was confirmed by microscopy of cultured cells 4 days upon the starting of the starving period (Figure $3 \mathrm{C}$ ).

hFGF-2 can execute its biological activities from outside the target cell by canonical receptor-based tyrosine kinase signalling or upon internalization and nuclear uptake [20], as this factor combines paracrine and nuclear activities in its biological function [31]. ArtlBs, as their natural counterparts, can penetrate mammalian cells upon exposure in absence of any intrinsic cytotoxicity $[13,18]$. In an attempt to evaluate the potential delivery of hFGF-2 from chemically pure ArtIBs, we determined the amount of protein released to the media upon incubation of the protein granules under physiological conditions. As observed, a significant portion of the growth factor was released from the insoluble hFGF-2 artificial material and detectable in the soluble fraction of the ArtIBs suspension, at $24 \mathrm{~h}$ of incubation (Figure 4A). Protein release was specifically notable in ArtIBs $\mathrm{Zn}^{+2}$ and ArtIBs $\mathrm{Ca}^{+2}$, while it was hardly detectable in the case of ArtlBs $\mathrm{Mn}^{+2}$. These release kinetics would be especially helpful in assisting the design of specific applications for those materials when intended for protein depots with time-prolonged supply of the contained growth factors. When cultured cells were exposed to such soluble fraction containing ArtIBs-released protein, stimulation of cell proliferation over the control, non-treated cells (100\%) was specially apparent when using ArtIBs Zn-solubilized protein, and at decreasing extents with ArtIBs formed with the assistance of $\mathrm{Ca}^{2+}$ and $\mathrm{Mn}^{2+}$ (Figure 4B). This is in agreement with the release kinetics shown in Figure 4A in which $\mathrm{Mn}$-based materials were less able to deliver the growth factor than the other ArtIB variants, indicative of a tighter clustering induced by this particular cation. At that point, we also investigated the conformational quality of the growth factor once removed from the particles. Importantly, the intrinsic fluorescence caused by exciting the protein with $295 \mathrm{~nm}$ ultraviolet light revealed no changes in the thermal profile of hFGF-2 released from ArtIBs. However, the CSM values at $25 \circ \mathrm{C}$ 
indicated a subtle change, suggesting conformational differences between the released proteins in comparison to the soluble version. Those results demonstrated a stability similar, if not higher, of the released protein compared to the original soluble hFGF-2

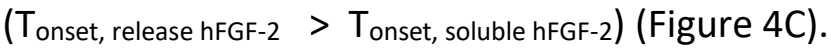

This set of results confirmed the correct selection of $\mathrm{Zn}^{2+}$ as default building ion, but also revealed that ArtIBs are good secretion materials, since the forming protein is progressively released without an immediate or a fast disintegration of the whole bulk material. These data also confirm the biological (and therefore structural) stability of hFGF-2 when submitted to packaging as ArtIBs and upon the subsequent release from them. Why some cations seem to favour better than others protein release, is an issue that remains to be solved. In this regard, protein release is inversely related to the binding strength between each cation-protein interaction. Distinct binding constants (Kb) have been demonstrated for $\mathrm{Cu}^{2+}$ and $\mathrm{Ca}^{2+}$ towards -synuclein [32]. Also, the structure-function effect of a particular cation on a given protein might be also and unique, as demonstrated by $\mathrm{Cu}^{2+} \mathrm{t} h$ a t a c t i v a t e p r o- a n g i fibroblast growth factor 2 [33]. Irrespective of that the precise mechanics of the observed differences, $\mathrm{Zn}^{2+}, \mathrm{Ca}^{2+}$ and $\mathrm{Mn}^{2+}$ (and other divalent cations) are all present at important amounts in the human body [34-37] and the clinical use of materials containing any of them would be completely plausible.

In vitro wound healing models are convenient tools to quickly evaluate the therapeutic potential of growth factors [38], and they have been specifically applied to examine the regenerative potential of the axolotl lipoxygenase administered as bacterial IBs [12]. A similar wound model (Figure 5A) was applied to scrutinize the activity of hFGF-2 ArtlBs $\mathrm{Zn}$, compared to the soluble version of the growth factor. As observed (Figure 5B,C), hFGF-2 ArtIBs Zn were more efficient than the soluble protein version in expanding the wound closure area, while the non-functional GFP ArtIBs Zn had only mild positive effects. These data, apart from being promising regarding the potential use of ArtIBs as carriers of growth factors, strongly suggest that the administration of hFGF-2 from ArtlBs could be more efficient than that of the soluble protein version (see also the supporting data in Figure 3A). This is probably associated to the depot effect of the protein granules, which extend the bioavailability of the growth factor for a longer time than when the 
protein is supplied in the soluble conventional form. The slow protein release from the material (Figure 4), that appears to be very stable, can of course account for this effect. ArtlBs, as fully proved here, can release plain soluble and functional protein for a biological role, in absence of bacterial contaminants that like chaperones, are expected to contribute to protein release from natural IBs because of their disaggregating activities [39, 40]. The amount of released protein is enough for enhanced cell proliferation (Figure 3A) and prolonged cell survival under starving conditions (Figure 3 $B, C)$, and this material favoured wound healing in an in vitro wound healing model (Figure 5). Such biological effects are superior than those obtained when exposing cells to either conventional IBs or soluble growth factor, supporting the further development of ArtIBs as a tremendously interesting biomaterial. The main relevance of ArtIBs is based on their pure composition. In contrast with the extremely heterogeneous nature of natural IBs, that contain cell wall components, nucleic acids, lipids and proteins as impurities from the producing cells $[19,24,41,42]$, these analogous artificial versions are uniquely formed by a single protein species assembled under defined physicochemical conditions. Being then chemically pure, synthetic protein depots are much less controversial regarding the potential toxicity of associated contaminants. The purification of soluble protein species (the building blocks for ArtIBs) is an scalable procedure at industrial level that has not posed any obstacle to the approval and generic clinical of recombinant proteins as drugs [43-45]. Therefore, the ArtIB packaging of recombinant protein drugs of human origin, namely enzymes, hormones, growth factors and others, irrespective of the cell factory used for the production of the soluble building blocks, should not envisage any problem related with immunotoxicity or poor biocompatibility. Therefore, ArtlBs would be functionally equivalent to functional secretory granules and applied in clinics in this context.

In addition, the properties of natural IBs are strongly dependent on the physiological conditions, nature and genetic background of the bacterial cell factory where they have been produced [46]. Among other observed influences, the culture conditions strongly affect the protein release capability [47], while the genetic background is a potent influencer of size, geometry, wettability, stiffness and other physicochemical parameters affecting IB performance $[48,49]$. In this context, ArtIBs benefit from the 
controlled fabrication process from purified protein, but also from the possibility to expand the source of the pure recombinant protein used for the generation of the materials. With a few exceptions, IBs are essentially produced in gram-negative bacteria, and therefore, restricted to non-glycosylated proteins [17], the polypeptides used for ArtIB fabrication can be obtained from bioproduction in any suitable cell factory, including yeast [50], plant [51] and mammalian cells [52], apart from any lessconventional microorganism or eukaryotic cells [50,53-56]. This fact largely expands the categories of proteins that can be packaged in form of secretory materials, including a large fraction of human proteins that are non-active or poorly active when produced in Escherichia coli [53].

As materials, ArtIBs are generated by a very easy fabrication process involving the protein itself and the linking ion, in mild environment-friendly conditions, that results in peculiar protein microparticles in which the unique forming protein shows a dual role as a scaffold and functional agent. The non-toxic amyloid composition of ArtIBs [18], that appears to be similar to that of natural IBs [57] and comparable to that of endocrine secretory granules $[7,9]$, confers mechanical stability and a protein network that engulfs or traps functional versions of the same protein. Protein release from ArtIBs can be then observed as a slow disintegration process during which building blocks of the material are progressively released from it, in a rate potentially regulatable by the divalent cation used in the fabrication. A similar protein release process supported by $\mathrm{Zn}^{2+}$ has been recently described for the natural secretion of the human growth hormone [58]. Being chemically pure, the further development of ArtIBs as storage/delivery micron sized granules for growth factors and other functional proteins should not face the constricting regulatory issues imposed to the chemically heterogeneous bacterial IBS and it opens a wide spectrum of possibilities for the controlled delivery of therapeutic proteins in many in vivo clinical contexts.

\section{Acknowledgements}

We are indebted to Agencia Estatal de Investigación (AEI) and to Fondo Europeo de Desarrollo Regional (FEDER) (grant PID2019-105416RB-I00 to EV and grant BIO2016- 
76063-R to AV, AEI/FEDER, UE) and AGAUR (2017SGR-229, to AV). We are also indebted to the Networking Research Center on Bioengineering, Biomaterials and Nanomedicine (CIBER-BBN) that is an initiative funded by the VI National R\&D\&I Plan 2008-2011, Iniciativa Ingenio 2010, Consolider Program, CIBER Actions and financed by the Instituto de Salud Carlos III, with assistance from the European Regional Development Fund. Protein production has been parti al l y per specifically by the Protein Production Platform of CIBER in Bioengineering, Biomaterials \& Nanomedicine (CIBER-BBN)/ IBB, at the UAB sePBioEs scientific-technical service (http://www.nanbiosis.es/portfolio/u1-protein-production-platform-ppp/) and the particle size analysis by the Biomaterial Processing and Nanostructuring Unit. Electron microscopy studies were performed by the Servei de Microscòpia at the UAB. Cell culture experiments were performed at the Cell Culture Service at the UAB (SCAC). AV received an ICREA ACADEMIA award. HLL and LSG supported by predoctoral fellowships from AGAUR (2019 FI_B_00352) and (2018FI_B2_00051), respectively.

\section{CRediT author statement}

Naroa Serna Investigation Writing - Review \& Editing Methodology Formal analysis

Olivia Cano-Garrido Investigation Writing - Review \& Editing

Julieta M. Sánchez Investigation Writing - Review \& Editing Formal analysis

Alejandro Sánchez-Chardi Data curation Writing - Review \& Editing Formal analysis

Laura Sánchez-García Investigation Writing - Review \& Editing

Hèctor López-Laguna Investigation Writing - Review \& Editing

Ester Fernández Conceptualization Writing - Review \& Editing

Esther Vázquez Conceptualization Writing - Review \& Editing Supervision

Antonio Villaverde Conceptualization Writing - Original Draft Funding acquisition Supervision 

References

[1] Gilmore KA, Lampley MW, Boyer C, Harth E. Matrices for combined delivery of proteins and synthetic molecules. Advanced drug delivery reviews. 2016;98:77-85.

[2] Li J, Mooney DJ. Designing hydrogels for controlled drug delivery. Nature reviews Materials. 2016;1.

[3] Hasnain MS, Nayak AK, Singh M, Tabish M, Ansari MT, Ara TJ. Alginate-based bipolymericnanobioceramic composite matrices for sustained drug release. International journal of biological macromolecules. 2016;83:71-7.

[4] Bolhassani A. Improvements in chemical carriers of proteins and peptides. Cell biology international. 2019;43:437-52.

[5] Cross LM, Carrow JK, Ding X, Singh KA, Gaharwar AK. Sustained and Prolonged Delivery of Protein Therapeutics from Two-Dimensional Nanosilicates. ACS applied materials \& interfaces. 2019;11:6741-50.

[6] Kapoor S, Kundu SC. Silk protein-based hydrogels: Promising advanced materials for biomedical applications. Acta biomaterialia. 2016;31:17-32.

[7] Maji SK, Perrin MH, Sawaya MR, Jessberger S, Vadodaria K, Rissman RA, et al. Functional amyloids as natural storage of peptide hormones in pituitary secretory granules. Science. 2009;325:328-32.

[8] Badtke MP, Hammer ND, Chapman MR. Functional amyloids signal their arrival. Science signaling. 2009;2:pe43.

[9] Mankar S, Anoop A, Sen S, Maji SK. Nanomaterials: amyloids reflect their brighter side. Nano reviews. 2011;2.

[10] de Marco A, Ferrer-Miralles N, Garcia-Fruitos E, Mitraki A, Peternel S, Rinas U, et al. Bacterial inclusion bodies are industrially exploitable amyloids. FEMS microbiology reviews. 2019;43:5372.

[11] Singhvi P, Saneja A, Srichandan S, Panda AK. Bacterial Inclusion Bodies: A Treasure Trove of Bioactive Proteins. Trends in biotechnology. 2020.

[12] Stamm A, Strauss S, Vogt P, Scheper T, Pepelanova I. Positive in vitro wound healing effects of functional inclusion bodies of a lipoxygenase from the Mexican axolotl. Microbial cell factories. 2018;17:57.

[13] Vazquez E, Corchero JL, Burgueno JF, Seras-Franzoso J, Kosoy A, Bosser R, et al. Functional inclusion bodies produced in bacteria as naturally occurring nanopills for advanced cell therapies. Advanced materials. 2012;24:1742-7.

[14] Liovic M, Ozir M, Zavec AB, Peternel S, Komel R, Zupancic T. Inclusion bodies as potential vehicles for recombinant protein delivery into epithelial cells. Microbial cell factories. 2012;11:67.

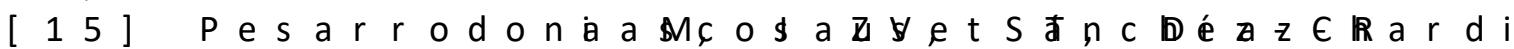

$\mathrm{J}$, et al. Targeting Antitumoral Proteins to Breast Cancer by Local Administration of Functional Inclusion Bodies. Advanced science. 2019; https://doi.org/10.1002/advs.201900849.

[16] Cespedes MV, Cano-Garrido O, Alamo P, Sala R, Gallardo A, Serna N, et al. Engineering Secretory Amyloids for Remote and Highly Selective Destruction of Metastatic Foci. Advanced materials. 2020;32:1907348.

[17] Villaverde A, Garcia-Fruitos E, Rinas U, Seras-Franzoso J, Kosoy A, Corchero JL, et al. Packaging protein drugs as bacterial inclusion bodies for therapeutic applications. Microbial cell factories. 2012;11:76.

[18] Sánchez J, López-Laguna H, Álamo P, Serna N, Sánchez-Chardi A, Nolan V, et al. Artificial inclusion bodies for clinical development Advanced science. 2020;7:1902420. 
[19] Rinas U, Hoffmann F, Betiku E, Estape D, Marten S. Inclusion body anatomy and functioning of chaperone-mediated in vivo inclusion body disassembly during high-level recombinant protein production in Escherichia coli. Journal of biotechnology. 2007;127:244-57.

[20] Seras-Franzoso J, Peebo K, Garcia-Fruitos E, Vazquez E, Rinas U, Villaverde A. Improving protein delivery of fibroblast growth factor- 2 from bacterial inclusion bodies used as cell culture substrates. Acta biomaterialia. 2014;10:1354-9.

[21] Sanchez JM, Sanchez-Garcia L, Pesarrodona M, Serna N, Sanchez-Chardi A, Unzueta U, et al. Conformational Conversion during Controlled Oligomerization into Nonamylogenic Protein Nanoparticles. Biomacromolecules. 2018;19:3788-97.

[22] Ferrao-Gonzales AD, Souto SO, Silva JL, Foguel D. The preaggregated state of an amyloidogenic protein: hydrostatic pressure converts native transthyretin into the amyloidogenic state. Proceedings of the National Academy of Sciences of the United States of America. 2000;97:6445-50.

[23] Cespedes MV, Fernandez Y, Unzueta U, Mendoza R, Seras-Franzoso J, Sanchez-Chardi A, et al. Bacterial mimetics of endocrine secretory granules as immobilized in vivo depots for functional protein drugs. Scientific reports. 2016;6:35765.

[24] Rinas U, Bailey JE. Protein compositional analysis of inclusion bodies produced in recombinant Escherichia coli. Applied microbiology and biotechnology. 1992;37:609-14.

[25] Carrio MM, Villaverde A. Localization of chaperones DnaK and GroEL in bacterial inclusion bodies. Journal of bacteriology. 2005;187:3599-601.

[26] Allen SP, Polazzi JO, Gierse JK, Easton AM. Two novel heat shock genes encoding proteins produced in response to heterologous protein expression in Escherichia coli. Journal of bacteriology. 1992;174:6938-47.

[27] Thomas JG, Baneyx F. Roles of the Escherichia coli small heat shock proteins IbpA and IbpB in thermal stress management: comparison with ClpA, ClpB, and HtpG in vivo. Journal of bacteriology. 1998;180:5165-72.

[28] Mogk A, Deuerling E, Vorderwulbecke S, Vierling E, Bukau B. Small heat shock proteins, ClpB and the DnaK system form a functional triade in reversing protein aggregation. Mol Microbiol. 2003;50:585-95.

[29] Matuszewska M, Kuczynska-Wisnik D, Laskowska E, Liberek K. The small heat shock protein IbpA of Escherichia coli cooperates with IbpB in stabilization of thermally aggregated proteins in a disaggregation competent state. Journal of Biological Chemistry. 2005;280:12292-8.

[30] Sanchez JM, Lopez-Laguna H, Alamo P, Serna N, Sanchez-Chardi A, Nolan V, et al. Artificial Inclusion Bodies for Clinical Development. Advanced science. 2020;7:1902420.

[31] Forthmann B, Grothe C, Claus P. A nuclear odyssey: fibroblast growth factor-2 (FGF-2) as a regulator of nuclear homeostasis in the nervous system. Cell Mol Life Sci. 2015;72:1651-62.

[32] Han JY, Choi TS, Kim HI. Molecular Role of $\mathrm{Ca}(2+)$ and Hard Divalent Metal Cations on Accelerated Fibrillation and Interfibrillar Aggregation of alpha-Synuclein. Scientific reports. 2018;8:1895.

[33] Stelling MP, Motta JM, Mashid M, Johnson WE, Pavao MS, Farrell NP. Metal ions and the extracellular matrix in tumor migration. The FEBS journal. 2019;286:2950-64.

[34] Maret W. Zinc biochemistry: from a single zinc enzyme to a key element of life. Advances in nutrition. 2013;4:82-91.

[35] Leonhard MJ, Chang ET, Loccisano AE, Garry MR. A systematic literature review of epidemiologic studies of developmental manganese exposure and neurodevelopmental outcomes. Toxicology. 2019;420:46-65.

[36] Fijorek K, Puskulluoglu M, Tomaszewska D, Tomaszewski R, Glinka A, Polak S. Serum potassium, sodium and calcium levels in healthy individuals - literature review and data analysis. Folia medica Cracoviensia. 2014;54:53-70.

[37] Glasdam SM, Glasdam S, Peters GH. The Importance of Magnesium in the Human Body: A Systematic Literature Review. Advances in clinical chemistry. 2016;73:169-93. 
[38] Guerra A, Belinha J, Jorge RN. Modelling skin wound healing angiogenesis: A review. J Theor Biol. 2018;459:1-17.

[39] Weibezahn J, Bukau B, Mogk A. Unscrambling an egg: protein disaggregation by AAA+ proteins. Microbial cell factories. 2004;3:1.

[40] Weibezahn J, Schlieker C, Tessarz P, Mogk A, Bukau B. Novel insights into the mechanism of chaperone-assisted protein disaggregation. Biological chemistry. 2005;386:739-44.

[41] Neubauer PF, B; Lilie, H; Villaverde, A; . Protein inclusion bodies in recombinant bacteria: Springer; 2006.

[42] Jurgen B, Breitenstein A, Urlacher V, Buttner K, Lin H, Hecker M, et al. Quality control of inclusion bodies in Escherichia coli. Microbial cell factories. 2010;9:41.

[43] Sanchez-Garcia L, Martin L, Mangues R, Ferrer-Miralles N, Vazquez E, Villaverde A. Recombinant pharmaceuticals from microbial cells: a 2015 update. Microbial cell factories. 2016;15:33.

[44] Lagasse HA, Alexaki A, Simhadri VL, Katagiri NH, Jankowski W, Sauna ZE, et al. Recent advances in (therapeutic protein) drug development. F1000Research. 2017;6:113.

[45] Walsh G. Pharmaceutical biotechnology products approved within the European Union. European journal of pharmaceutics and biopharmaceutics : official journal of Arbeitsgemeinschaft fur Pharmazeutische Verfahrenstechnik eV. 2003;55:3-10.

[46] Garcia-Fruitos E, Vazquez E, Diez-Gil C, Corchero JL, Seras-Franzoso J, Ratera I, et al. Bacterial inclusion bodies: making gold from waste. Trends in biotechnology. 2012;30:65-70.

[47] Peternel S, Grdadolnik J, Gaberc-Porekar V, Komel R. Engineering inclusion bodies for non denaturing extraction of functional proteins. Microbial cell factories. 2008;7:34.

[48] Diez-Gil C, Krabbenborg S, Garcia-Fruitos E, Vazquez E, Rodriguez-Carmona E, Ratera I, et al. The nanoscale properties of bacterial inclusion bodies and their effect on mammalian cell proliferation. Biomaterials. 2010;31:5805-12.

[49] Garcia-Fruitos E, Seras-Franzoso J, Vazquez E, Villaverde A. Tunable geometry of bacterial inclusion bodies as substrate materials for tissue engineering. Nanotechnology. 2010;21:205101.

[50] Mattanovich D, Branduardi P, Dato L, Gasser B, Sauer M, Porro D. Recombinant protein production in yeasts. Methods in molecular biology. 2012;824:329-58.

[51] Rozov SM, Deineko EV. [Strategies for Optimizing Recombinant Protein Synthesis in Plant Cells: Classical Approaches and New Directions]. Molekuliarnaia biologiia. 2019;53:179-99.

[52] Barnes LM, Dickson AJ. Mammalian cell factories for efficient and stable protein expression. Current opinion in biotechnology. 2006;17:381-6.

[53] Corchero JL, Gasser B, Resina D, Smith W, Parrilli E, Vazquez F, et al. Unconventional microbial systems for the cost-efficient production of high-quality protein therapeutics. Biotechnology advances. 2013;31:140-53.

[54] Rebello S, Abraham A, Madhavan A, Sindhu R, Binod P, Karthika Bahuleyan A, et al. Nonconventional yeast cell factories for sustainable bioprocesses. FEMS microbiology letters. 2018;365.

[55] Song AA, In LLA, Lim SHE, Rahim RA. A review on Lactococcus lactis: from food to factory. Microbial cell factories. 2017;16:55.

[56] Vieira Gomes AM, Souza Carmo T, Silva Carvalho L, Mendonca Bahia F, Parachin NS. Comparison of Yeasts as Hosts for Recombinant Protein Production. Microorganisms. 2018;6.

[57] Cano-Garrido O, Rodriguez-Carmona E, Diez-Gil C, Vazquez E, Elizondo E, Cubarsi R, et al. Supramolecular organization of protein-releasing functional amyloids solved in bacterial inclusion bodies. Acta biomaterialia. 2013;9:6134-42.

[58] Jacob RS, Das S, Ghosh S, Anoop A, Jha NN, Khan T, et al. Amyloid formation of growth hormone in presence of zinc: Relevance to its storage in secretory granules. Scientific reports. 2016;6:23370. 
[59] Vazquez E, Roldan M, Diez-Gil C, Unzueta U, Domingo-Espin J, Cedano J, et al. Protein nanodisk assembling and intracellular trafficking powered by an arginine-rich (R9) peptide. Nanomedicine. 2010;5:259-68.

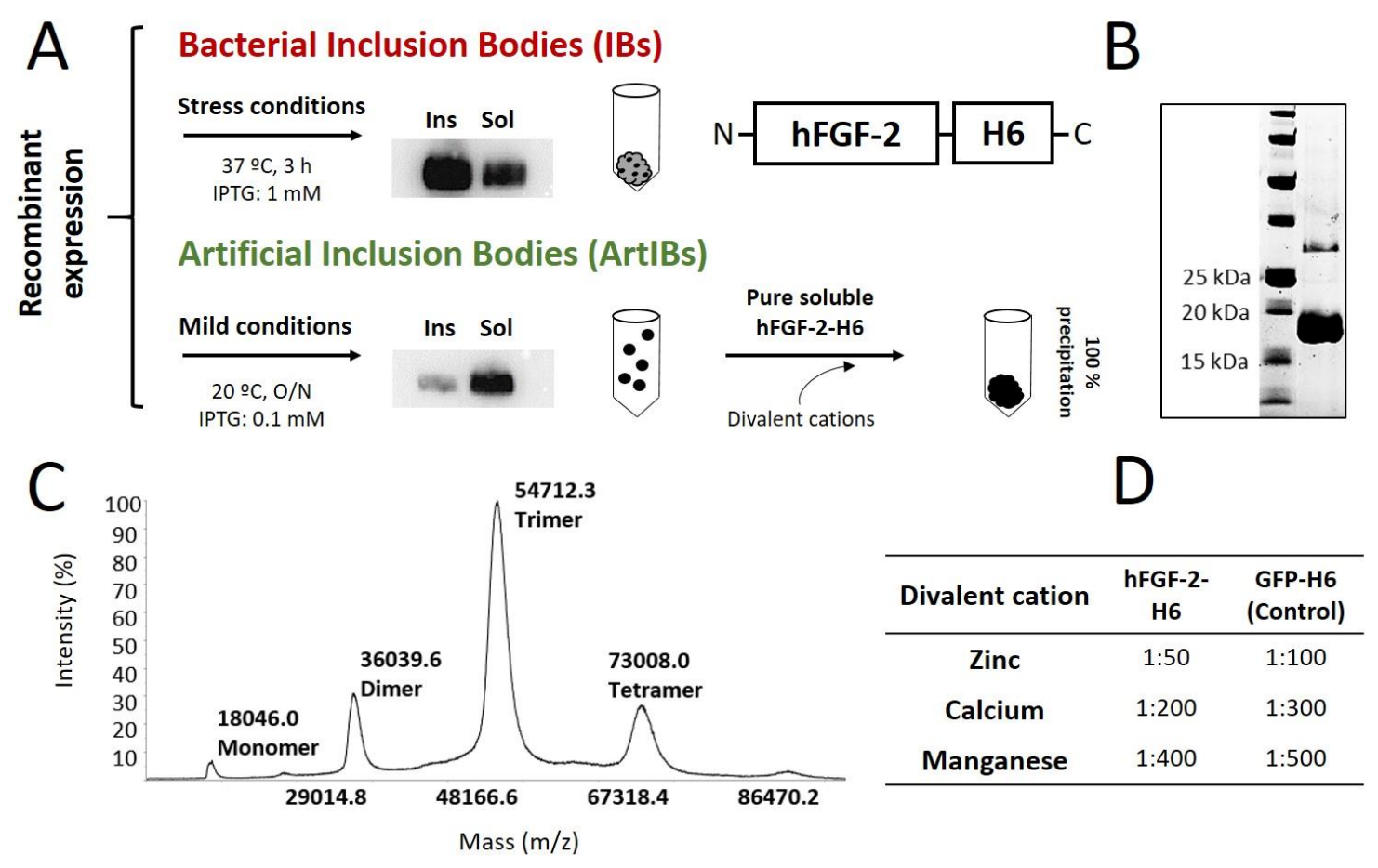

Figure 1. Production of bacterial IBs and ArtlBs based on hFGF-2. A. The modular scheme of hFGF-2-H6 is shown, indicating the amino (N) and carboxy (C) terminal ends. Note that sizes of the boxes are only approximate. GFP-H6 has the same modular scheme in which hFGF-2 is replaced by GFP [59]. The recombinant protein was produced in E. coli under a set of mild or stressful conditions (indicated), that resulted in a different fractioning between soluble (Sol) and insoluble (Ins) protein fractions (shown here by anti-H6 Western blots). Natural IBs were straightforward recovered from the insoluble protein fraction produced under stress conditions. Purified soluble protein obtained under mild production conditions was used to fabricate ArtIBs as described [18]. B. The hFGF-2 soluble protein sample submitted to denaturing SDS-PAGE and further TGX protein staining, revealing the integrity of the protein $(18 \mathrm{kDa})$ and the absence of degradation bands. The molecular weight of markers (left line) is indicated. C. MALDITOF analysis of the same hFGF-2 sample, indicating different oligomeric states of the protein in its native state. D. Molar ratio (protein:cation) of the tested divalent cations that resulted in $100 \%$ of ArtIB formation in hFGF-2-H6 and the control protein GFP-H6. 

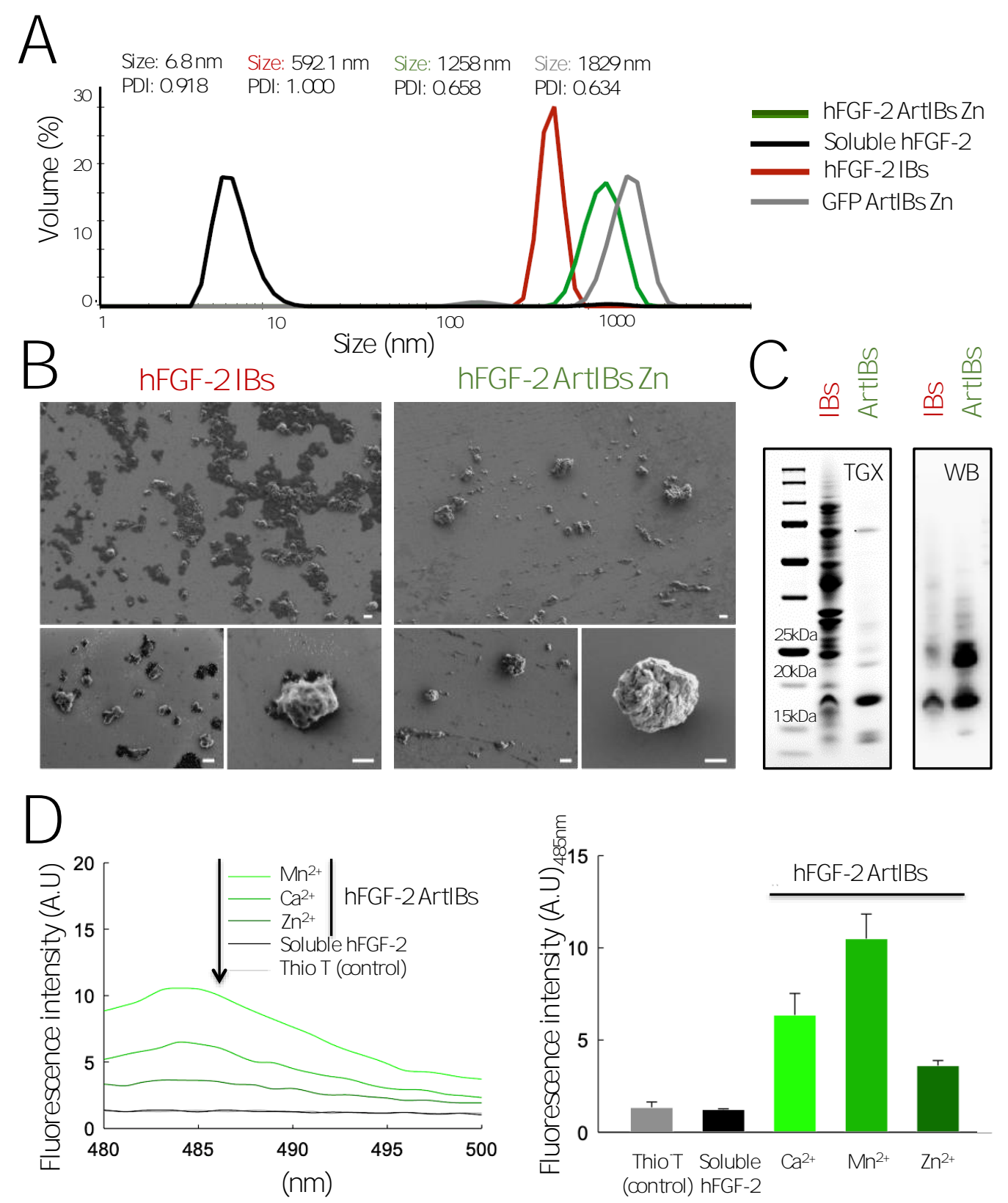

Figure 2. Characterization of hFGF-2 IBs and hFGF-2 ArtIBs. A. Hydrodynamic size of hFGF-2 IBs and hFGF-2 ArtIBs based on $\mathrm{Zn}^{2+}$. Numbers indicate the peak of the DLS plot (size) and the polydispersion index (PDI). GFP-H6 ArtIBs $\mathrm{Zn}$ and soluble hFGF-2 were used as control. B. Representative FESEM images at three magnifications showing from broad fields to IBs detail of isolated hFGF-2 IBs and hFGF-2 ArtIBs Zn. Bars indicate: 500 $\mathrm{nm}$. C. SDS-PAGE and further TGX protein staining or Western blot immunodetection of both hFGF-2 bacterial IBs and hFGF-2 ArtIBs based on $\mathrm{Zn}^{2+}$. The molecular weight of markers is also indicated. The migration of the soluble protein is shown in Figure 1 B. D. Thio $T$ fluorescence emission spectra recorded at $e_{x}=450 \mathrm{~nm}$. Left: Thio $T$ spectra of hFGF-2 ArtlBs indicated by its cationic composition. The control represents the fluorescence of Thioflavin $\mathrm{T}$ in carbonate buffer. Thio $\mathrm{T}$ interacting with soluble hFGF-2 was also included. Right: Thio T fluorescence emission intensity at $485 \mathrm{~nm}$. 


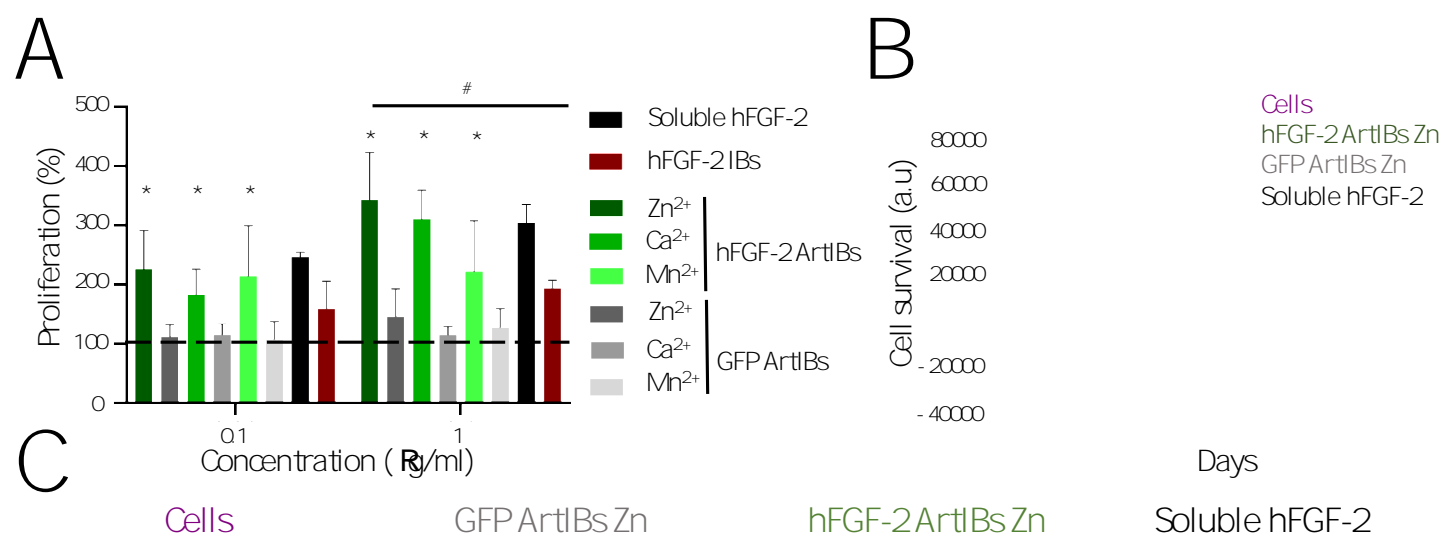

Figure 3. Biological effects of hFGF-2 ArtIBs. A. Proliferation of NIH3T3 cells cultured on

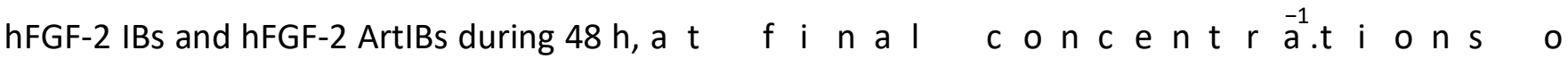
GFP-H6 ArtIBs were used as negative control and soluble hFGF-2 as positive control. Significant differences with the respective GFP ArtIB controls are indicated ( $\left.{ }^{*} p<0.01\right)$. Differences between hFGF-2 ArtIBs and hFGF-2 bacterial IBs are also indicated (\# $p<0.05)$. B. Survival of NIH3T3 cells cultured on starvation medium containing $1 \%$ FBS in the presence of $1 \mu \mathrm{g}^{-1}$ of $\mathrm{mFFGF-2}$ ArtIBs Zn, GFP-H6 ArtIBs Zn and soluble hFGF-2. Data within upper and lower curves are significantly different to each other in the interval from day 2 to day $6(p<0.01)$.C. Representative light microscopy images of cells at day 4 of the cell survival assay showing the morphological changes induced by hFGF2 ArtIBs $\mathrm{Zn}$ and soluble hFGF-2. Bars indicate $50 \mu \mathrm{m}$. 


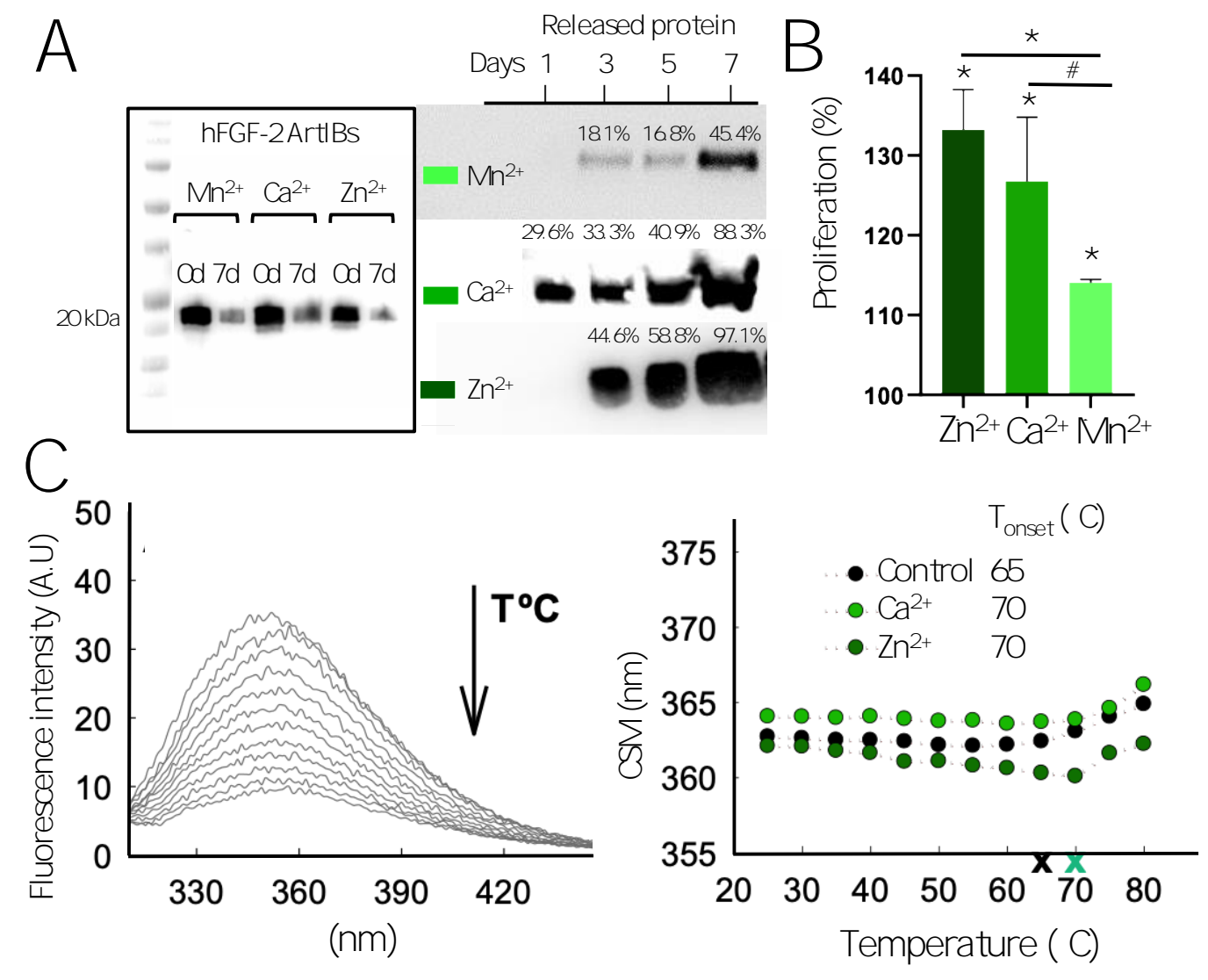

Figure 4. Release of soluble protein from hFGF-2 ArtlBs. A. Western blot detection of hFGF-2 ArtIBs (formed by alternative cations) before and after incubation at 37으 for 7 days. Western blot detection and the percentage of the released soluble protein after incubation of those ArtIBs at 37으 $\mathrm{C}$ for 1, 3, 5 and 7 days is also shown. B. Proliferation of NIH3T3 cells treated with the soluble hFGF-2 released from hFGF-2 ArtlBs during $48 \mathrm{~h}$. The cross-linking cations are indicated in each case. The symbols indicate significant differences ( $* p<0.01, \# p<0.05$ ) with untreated cells or between cells treated with released protein from diverse materials. C. Thermal stability of released soluble hFGF-2 from ArtIBs. Left: Typical intrinsic fluorescence spectra of soluble hFGF-2 recorded at different temperatures. The arrow indicates the heating direction. Right: CSM vs temperature. The black cross indicates the onset temperature ( $\left.T_{\text {onset }}\right)$ of the soluble protein and the green cross indicates the $T_{\text {onset }}$ of the protein released from ArtIBs. 


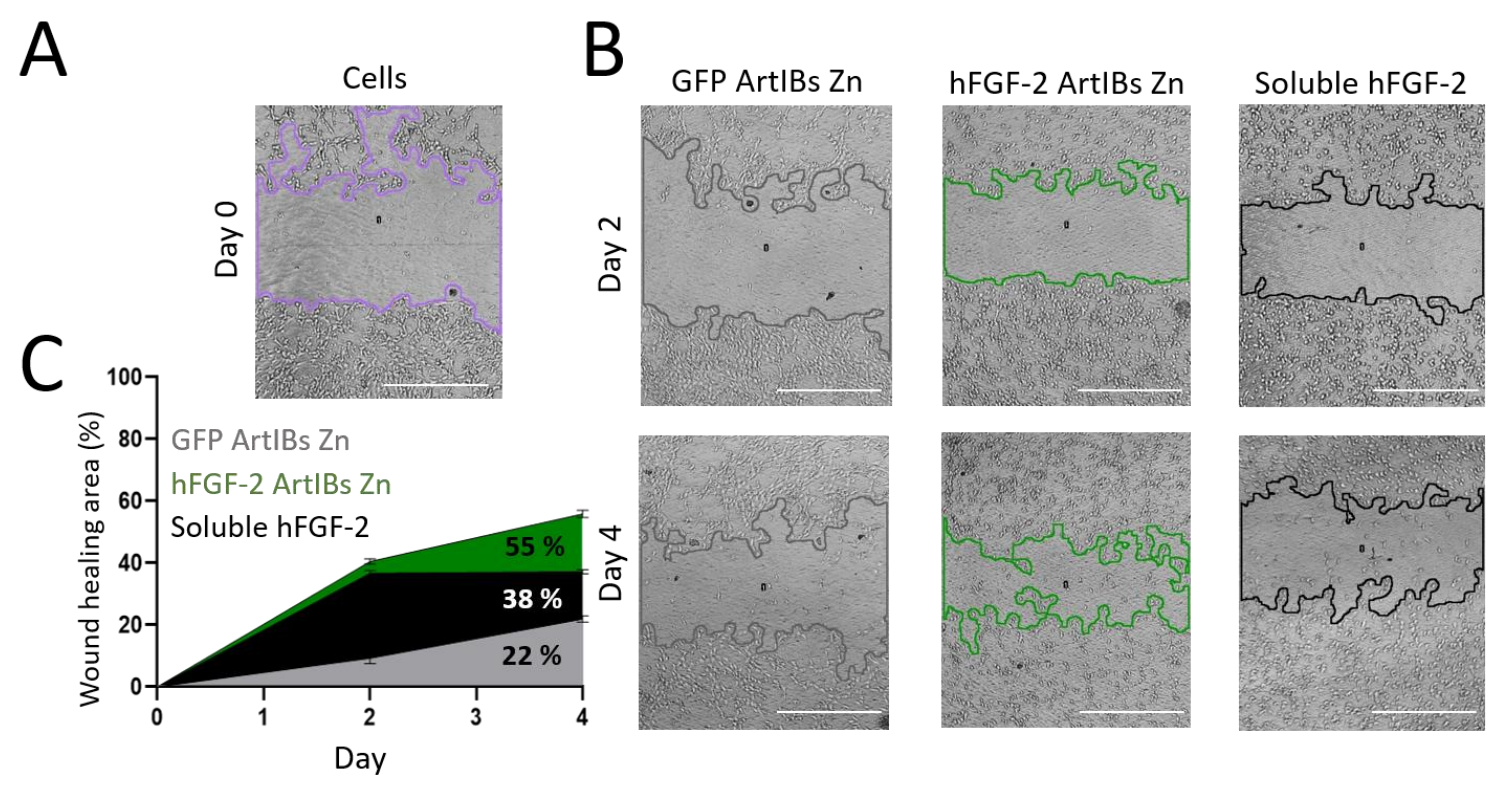

Figure 5. Wound healing activity. A. Measurement of wound area after culture-insert removal. B. Measurement of wound areas coating with $1 \mu \mathrm{g}^{-1}$ of imAGF-2 ArtIBs Zn, negative control GFP-H6 ArtIBs $\mathrm{Zn}$ and treatment with positive control, soluble hFGF-2. Bars indicate $50 \mu \mathrm{m}$. All measurements were done using the MRI wound healing tool macro for ImageJ. C. Wound closure area \% determined in the wound healing assay after 2 and 4 days. On the last day of the experiment, the differences in these areas are significantly different between soluble hFGF-2 and GFP ArtIB $(p<0.01)$, and between hFGF-2 ArtIB and GFP ArtIB ( $p<0.01)$. Wound closure area is also significantly different between soluble hFGF-2 and hFGF-2 ArtIB $(p<0.05)$. 